\title{
Gender differences in healthcare service utilisation 1 year before suicide: national record linkage study
}

Chia-Ming Chang, Shih-Cheng Liao, Hung-Chi Chiang, Ying-Yeh Chen, Kwan-Cho Tseng,

Yeuk-Lun Chau, Hsiu-Ju Chang and Ming-Been Lee

\section{Summary}

All suicides ( $n=12497)$ in Taiwan in 2001-2004 were identified from mortality records retrieved from the National Health Insurance Database. Altogether, $95.1 \%$ of females and $84.9 \%$ of males had been in contact with healthcare services in the year before their death. Females received significantly more diagnoses of psychiatric disorders (48.0\% v. 30.2\%) and major depression $(17.8 \%$ v. $7.4 \%)$ than males. Such differences were consistent across different medical settings where contact with hospital-based non-psychiatric physicians was as common as with general practitioners (GPS). However, diagnoses of psychiatric disorders were underdiagnosed in both genders.

\section{Declaration of interest}

None.
Previous studies ${ }^{1-4}$ have shown that most people who die by suicide have contact with healthcare services and have suffered mental disorders (particularly major depression) prior to the event. $^{3-5}$ A few studies ${ }^{4-6}$ have demonstrated that females are more likely than males to contact healthcare services before taking their own lives. However, the diagnoses they receive and the association of these diagnoses with medical settings are unknown.

These issues are important for suicide prevention. With the recent increase of suicide in Taiwan, the expertise of physicians and their diagnoses may have implications for the management of suicide prevention in different medical settings. We investigated the gender differences in healthcare service utilisation and diagnoses received by patients 1 year prior to suicide.

\section{Method}

Deaths were identified as suicides between 1 January 2001 and 31 December 2004 using the ICD-9 ${ }^{7}$ codes E950-E959. To determine healthcare service utilisation in the year preceding death, the record of each suicide case was retrieved from the National Health Insurance Database (NHID; www.nhri.org.tw/nhird/) using a personal identification number. Retrieval of data was performed and supervised by the Department of Health.

Non-mental-health physicians were classified as either hospitalbased non-psychiatric physicians or general practitioners (GPs). General practitioners included all physicians in private practice and those based in healthcare services not covered by the NHI system. The out-patient or in-patient codes ICD-9-CM 290.** 319.** were defined as psychiatric disorders and ICD-9-CM $296.2^{*}-296.3^{\star}$ as major depression. ${ }^{8}$ The study was approved by the ethics committee of the National Taiwan University Hospital (NTUH-REC no. 200711030R).

Descriptive analyses were carried out using SAS version 9.1 for Windows. Chi-squared tests were used to compare the proportion of males and females who used healthcare services in the year prior to suicide. A probability level of $<0.05$ was considered significant.

\section{Results}

A total of 12497 patients died by suicide (8523 males, 3974 females; male-to-female ratio 2.1:1; mean age 49.2 years $($ s.d. $=18.0))$ in the 4 -year period. Overall, $88.2 \%$ had had at least one contact with healthcare services, but only $24.9 \%$ had had contact with psychiatrists in the year before their death. The most common non-psychiatric contacts were with hospital-based non-psychiatric physicians (79.5\%) and GPs (72.1\%). Only $35.9 \%$ of all patients who died by suicide received a diagnosis of psychiatric disorder in the 12 months prior to their death, and only $10.7 \%$ received a diagnosis of major depression (Table 1 ).

A higher proportion of females than males had contacted healthcare service providers, psychiatrists, hospital-based nonpsychiatric physicians, and GPs. Psychiatric disorders were diagnosed in $48 \%$ of females and $30.2 \%$ of males, but major depression was diagnosed in only $17.8 \%$ of females and $7.4 \%$ of males. Psychiatric disorders and major depression were diagnosed in significantly more female than male patients regardless of the medical setting. The $P$-values for all of the above were $<0.0001$.

\section{Discussion}

This study found that significantly more females than males had contacted healthcare services prior to suicide. These results are consistent with other findings. ${ }^{5,6}$ We also found that major depression was diagnosed in only $17.8 \%$ of females and $7.4 \%$ of males before their death. In addition, $15.1 \%$ of males and $4.9 \%$ of females had had no contact with services in the year prior to suicide. Our results show that mental disorders and major depression are largely underdiagnosed, especially in males. Possibly, underdiagnosis of mental disorders and major depression in suicide cases reflects a cultural bias against confronting emotional/ psychological problems.

Healthcare systems and help-seeking behaviours are different between Taiwan and Western societies. ${ }^{9}$ A national study in the $\mathrm{UK}^{4}$ focused on suicides within 12 months of contact with mental health services. Our study demonstrated the utilisation of nonmental health services and found that contact with hospital-based non-psychiatric physicians was as frequent as with GPs. Hospitalbased non-psychiatric physicians seemed more likely than GPs to make a diagnosis of psychiatric disorder and major depression (the between-group differences were not analysed since these two groups were not mutually exclusive).

This study has some limitations. First, possible information biases in the NHID and mortality database may have affected 


\begin{tabular}{|c|c|c|c|}
\hline & $\begin{array}{c}\text { Males }(n=8523) \\
n(\%)\end{array}$ & $\begin{array}{c}\text { Females }(n=3974) \\
n(\%)\end{array}$ & $\begin{array}{c}\text { Total }(n=12497) \\
n(\%)\end{array}$ \\
\hline \multicolumn{4}{|l|}{ Healthcare contacts } \\
\hline Any & 7239 (84.9) & 3778 (95.1) & $11017(88.2)$ \\
\hline Psychiatrists & $1721(20.2)$ & $1383(34.8)$ & $3104(24.9)$ \\
\hline Hospital-based non-psychiatric physicians & $6400(75.1)$ & $3536(89.0)$ & $9936(79.5)$ \\
\hline General practitioners & $5652(66.3)$ & 3359 (84.5) & $9011(72.1)$ \\
\hline No contacts & $1284(15.1)$ & $196(4.9)$ & $1480(11.8)$ \\
\hline \multicolumn{4}{|l|}{ Psychiatric disorders diagnoses } \\
\hline Any & $2575(30.2)$ & $1907(48.0)$ & $4482(35.9)$ \\
\hline Psychiatrists & $1686(19.8)$ & $1362(34.3)$ & $3048(24.4)$ \\
\hline Hospital-based non-psychiatric physicians & $1326(15.6)$ & $972(24.5)$ & $2298(18.4)$ \\
\hline General practitioners & $596 \quad(7.0)$ & $547(13.8)$ & $1143 \quad(9.2)$ \\
\hline \multicolumn{4}{|l|}{ Major depression diagnoses } \\
\hline Any & $633(7.4)$ & $706(17.8)$ & $1339(10.7)$ \\
\hline Psychiatrists & $490 \quad(5.8)$ & $574(14.4)$ & 1064 \\
\hline Hospital-based non-psychiatric physicians & $184(2.2)$ & $213(5.4)$ & 397 (3.2) \\
\hline General practitioners & $85(1.0)$ & $94 \quad(2.4)$ & $179(1.4)$ \\
\hline
\end{tabular}

the results. One study ${ }^{10}$ suggested that open verdicts should be included in suicide research, and one study in Taiwan ranked the quality of death certification as fair: ${ }^{10}$ we did not include open verdicts in our analysis. Second, since the NHID was established for the purpose of healthcare provision and reimbursement, the reliability of the diagnoses cannot be confirmed. Third, there is no comparable information relating to non-suicide cases. Thus, whether contact with the healthcare system had any role in preventing suicide, for example by delaying it, cannot be determined. Further study should be performed to explore the causal association between healthcare contact, diagnoses, antidepressants and suicides.

In summary, our study found a female predominance in health service utilisation and in receiving a diagnosis of a mental disorder and major depression prior to suicide, although disorders were underdiagnosed in both genders. We suggest that hospitalbased non-psychiatric physicians and GPs should be better trained to identify suicide risk and treat depression to reduce the suicide rate in Taiwan.

Chia-Ming Chang, MD, PhD, Department of Psychiatry and Suicide Prevention Center, Chang Gung Memorial Hospital, and Chang Gung University, Lin-Ko, Taiwan; Shih-Cheng Liao, MD, MS, Department of Psychiatry, National Taiwan University Hospital; Hung-Chi Chiang, MPH, National Suicide Prevention Center; Ying-Yeh Chen, MD, PhD, Taipei City Psychiatric Centre, Taipei City; Kwan-Cho Tseng, MD, PhD, Department of Psychiatry, National Taiwan University Hospital; Yeuk-Lun Chau, MS, Department of Psychiatry and Suicide Prevention Center, Chang Gung Memorial Hospital, Lin-Ko; Hsiu-Ju Chang, PhD, RN, College of Nursing, Taipei Medical University; Ming-Been Lee, MD, Department of Psychiatry, National Taiwan University Hospital and College of Medicine, Taiwan National Suicide Prevention Center, Taiwan

Correspondence: Ming-Been Lee, Department of Psychiatry, National Taiwan University Hospital, No.7 Chung San South Road, Taipei, Taiwan. Email: mingbeen@ha.mc.ntu.edu.tw

First received 14 Apr 2008, final revision 2 Jun 2009, accepted 17 Jun 2009

\section{Funding}

This study was supported by a grant from the Department of Health, Executive Yuan, Taiwan, R.O.C. to the National Suicide Prevention Center (94-3084), and by a grant from the Department of Health, Executive Yuan, Taiwan, R.O.C. (DOH93-TD-M-113- 007) and National Science Council (NSC 93-2314-B-040-027) to C.-M.C.

\section{References}

1 Luoma JB, Martin CE, Pearson JL. Contact with mental health and primary care providers before suicide: a review of the evidence. Am J Psychiatry 2002; 159: 909-16.

2 Pirkis J, Burgess P. Suicide and recency of health care contacts. A systematic review. Br J Psychiatry 1998; 173: 462-74.

3 Cheng ATA. Mental illness and suicide: a case-control study in east Taiwan. Arch Gen Psychiatry 1995; 52: 594-603.

4 Appleby L, Shaw J, Amos T, McDonnell R, Harris C, McCann K, et al. Suicide within 12 months of contact with mental health services: national clinical survey. BMJ 1999; 318: 1235-9.

5 Isometsa ET, Aro HM, Henriksson MM, Heikkinen ME, Lonnqvist JK. Suicide in major depression in different treatment settings. J Clin Psychiatry 1994; 55: $523-7$.

6 Hawton K. Sex and suicide. Gender differences in suicidal behaviour. Br J Psychiatry 2000; 177: 484-5.

7 World Health Organization. International Classification of Diseases, Ninth Revision (ICD-9). WHO, 1978.

8 US Department of Health and Human Services. International Classification of Diseases, Ninth Revision, Clinical Modification (ICD-9-CM) (6th edn). US Department of Health and Human Services, 1998.

9 Chen TJ, Chou LF, Hwang SJ. Patterns of ambulatory care utilization in Taiwan. BMC Health Serv Res 2006; 6: 1-8.

10 Linsley KR, Schapira K, Kelly TP. Open verdict $v$. suicide - importance to research. Br J Psychiatry 2001; 178: 465-8.

$11 \mathrm{Lu}$ TH, Sun, SM, Huang SM, Lin JJ. Mind your manners: quality of manner of death certification among medical examiners and coroners in Taiwan. Am J Forensic Med Pathol 2006; 27: 352-4. 